\title{
Hepatic haematoma rupture in a Warmblood: a rare complication of hepatic AA amyloidosis
}

\author{
Max Diekstall', Juliane Veh? and Astrid B. M. Rijkenhuizen² \\ 'Pferdeklinik Leichlingen, Am Further Weiher 1, 42799 Leichlingen, Germany \\ 2 Veterinary Consultancy, Singel 10,3961 CE Wijk Bij Duurstede, The Netherlands
}

\begin{abstract}
Summary: A 15-year-old Warmblood gelding was referred to the clinic because of abdominal discomfort. One year before clinical evaluation, the horse presented with a mass in the back muscles that had first appeared 3 years prior. The mass, located in the caudal saddle position, was of an unknown cause and did not disturb the horse in any way. A calcification was recognized radiologically; however, treatment was not pursued because of high risk of poor wound healing and infection in this area. On the day of referral the horse was presenting signs of colic as a result of a haemoperitoneum following a ruptured haematoma in the liver, which was diagnosed by laparoscopy in the standing position. The horse was euthanized because of persistent, extensive haemorrhage from the unstoppable bleeding of the liver. At necropsy liver amyloidosis with subcapsular and intrahepatic haematoma was diagnosed. The calcified mass in the horse's back muscle was considered to be an old infected wound that could be the trigger for Amyloid-A (AA) amyloidosis. To the author's knowledge, this condition has not yet been described in horses.
\end{abstract}

Keywords: equine, haemoperitoneum, laparoscopy, amyloidosis, liver rupture

Citation: Diekstall M., Veh J., Rijkenhuizen A. B. M. (2019) Hepatic haematoma rupture in a Warmblood: a rare complication of hepatic AA amyloidosis. Pferdeheilkunde 35, 120-125; DOI 10.21836/PEM20190203

Correspondence: Prof. Astrid B. M. Rijkenhuizen, Veterinary Consultancy, Singel 10, 3961 CE Wijk Bij Duurstede, The Netherlands; a.rijkenhuizen@gmail.com

\section{Introduction}

Haemoperitoneum is an uncommon cause of anaemia by blood loss, and it is a potentially life-threatening condition for horses (Conwell et al. 2010). There are many causes of haemoperitoneum, such as splenic injury, rupture of neoplasia, postsurgical haemorrhage, coagulopathy, and bleeding from the reproductive tract with idiopathic origin (Green et al. 1988, Gatewood et al. 1990, Pusterla et al. 2005, Hawkins 2016). The major clinical signs are abdominal discomfort, dullness, anaemia and depression. For determining diagnosis, ultrasonography and abdominocentesis are the best minimally invasive ways to detect blood in the abdominal cavity. Furthermore, diagnostic laparotomy and standing laparoscopy are options for identifying the source of the haemorrhage (Conwell et al. 2010, Klohnen 2012). In the presented case, spontaneous rupture of hepatic haematoma with resultant intraperitoneal haemorrhage occurred as a consequence of liver AA amyloidosis. A calcified mass in the horse's back muscle was considered to be an old infected wound that could be responsible for the AA amyloidosis. The horse was euthanized because of the persistent, extensive haemorrhage that occurred due to the unstoppable bleeding of the liver. To the author's knowledge, this condition has not yet been described in horses.

\section{Case history}

A 15-year-old Warmblood gelding was presented to the equine clinic with a mass in the back muscles of the caudal saddle position, which had been present for at least 3 years and had not changed in size since it first appeared (Fig. 1).
The horse had no clinical problems; however, the owners wanted to know what the mass was. A hard, nonpainful swelling with a length of $18 \mathrm{~cm}$ and a width of $5 \mathrm{~cm}$ was located just under the skin and was pronounced $(2 \mathrm{~cm})$. The skin was attached to the mass, and the mass could not be moved in the muscles. No skin lesion or scar tissue could be found. The radiograph showed a radiopaque-chambered structure just under the skin. Ultrasonography indicated a non-echogenic mass, with a diameter of approximately $3-5 \mathrm{~cm}$, with clear fluid accumulation in the centre. Since the horse had no clinical problems or potential risk of infection of the mass, it was not punctured, and no treatment was initiated.

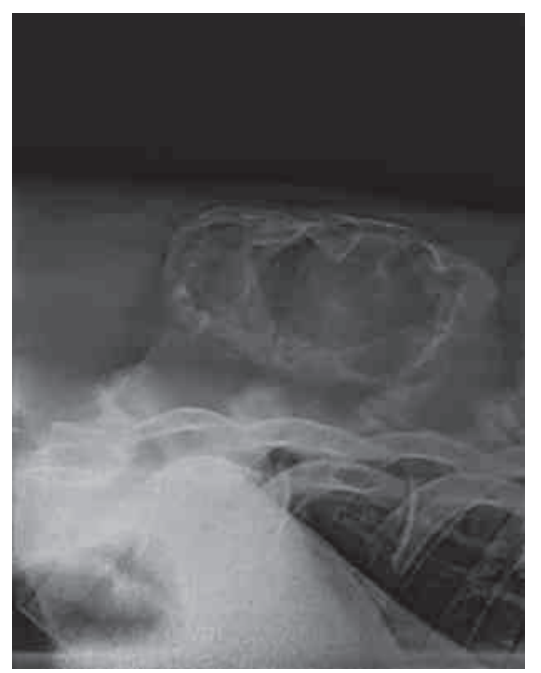

Fig. 1 X-ray of the mass in the back muscle of the horse. The front of the horse is on the left. 
One year later, the owners noticed a reduced general condition and reduced appetite for 2 weeks, and the horse then suddenly showed colic symptoms, such as pawing at the ground with the forelimb and lying down and looking towards the abdomen. The referring veterinarian noticed an increased heart rate (60 beats/min) and pale, dry mucous membranes of the mouth and conjunctiva. Abdominal auscultation on the right and left flanks indicated reduced borborygmi, and transrectal examination identified no abnormalities, besides a rectum full of faeces. The horse was treated symptomatically with $25 \mathrm{ml}$ scopolamine butylbromide $(5 \mathrm{ml} / 100 \mathrm{~kg})^{1}$ and 40 $\mathrm{ml}$ metamizol $(5 \mathrm{ml} / 100 \mathrm{~kg})^{2}$ intravenously. In the following hours, the condition of the horse did not improve and the horse was referred to the clinic.

\section{Clinical findings}

On presentation, the horse was lightly depressed and had a heart rate of 72 beats/min, a respiratory rate of 28 breaths/ min and a rectal temperature of $37.6^{\circ} \mathrm{C}$. The mucous membranes were pale, and the capillary refill time was 2 seconds. At abdominal auscultation of the right and left quadrants, reduced borborygmi were noticed. Transrectal examination indicated no abnormalities except that the rectum was filled with solid, clotted faeces. On ultrasonography of the ventral abdomen, a large amount of free echogenic fluid was detected. The fluid appeared clear without any signs of fibrin or blood clots. The liver had an inhomogeneous structure, with echogenic and anechogenic regions alternating completely over the organ on the right side of the abdomen. The intestine showed reduced motility; however, no gastrointestinal pathology was found. The peritoneal punctate obtained by abdominocentesis consisted of a $5 \mathrm{ml}$ clear, reddish liquid with a total protein concentration of $5.0 \mathrm{~g} / \mathrm{I}$ (reference range $[\mathrm{rr}]<12 \mathrm{~g} / \mathrm{l}$ ) and a lactate concentration of $1.2 \mathrm{mmol} / \mathrm{l}(\mathrm{rr}: 1.11-1.78 \mathrm{mmol} / \mathrm{l})$. Haematology indicated an erythrocyte count of $5.5 \times 10^{6}$ / $\mu \mathrm{l}$ (reference range $5.5-9.5 \times 10^{6} / \mu \mathrm{l}$ ), a packed cell volume [PCV] of $25.5 \%$ (rr" 30-47\%) and a haemoglobin content of $8.9 \mathrm{mmol} / \mathrm{l}$ ( $\mathrm{rr} 10.7-16.5 \mathrm{mmol} / \mathrm{l})$. The number of leukocytes was $14.1 \times 10^{3} / \mu \mathrm{l}\left(\mathrm{rr} 5.4-14.3 \times 10^{3} / \mu \mathrm{l}\right)$ with marked neutrophilia $\left(11.4 \times 10^{3} / \mu \mathrm{l}\right.$, rr $\left.2.3-8.6 \times 10^{3} / \mu \mathrm{l}\right)$. The number of monocytes was slightly increased $\left(1.1 \times 10^{3} / \mu \mathrm{l}, \mathrm{rr} 0-1 \times 10^{3} / \mu \mathrm{l}\right)$, and thrombocytes were within the normal limits $(95000 / \mu \mathrm{l})$.

Clinical chemistry indicated hyperglycaemia (glucose of 253 $\mathrm{mg} / \mathrm{dL}$ (rr: $75-115 \mathrm{mg} / \mathrm{dL}$ ), hypoproteinaemia (total protein of $48 \mathrm{~g} / \mathrm{l}(\mathrm{rr} \mathrm{52-79} \mathrm{g} / \mathrm{l})$, slightly increased blood urea nitrogen (BUN) (28 mg/dL, rr 10-24 mg/dL), and increased aspartate aminotransferase (AST) (382 IU/L, rr 226-366 IU/L), whereas all other parameters such as lactate, alkaline phosphatase (ALP), calcium (CA), creatinine (CREA), gamma glutamyl transferase (GGT) and lactase dehydrogenase enzyme (LDH) were within normal limits.

Based on the depressed behaviour of the horse, the pale mucous membrane, the decreased PCV and haemorrhagic abdominal fluid, blood loss into the abdominal cavity was suspected. The horse was hospitalized and underwent further monitoring every two hours (clinically and by PCV measurement) to observe if the suspected blood loss resolved spontaneously. $\mathrm{NaCl} 0.9 \%{ }^{3}$ at a rate of $3 \mathrm{~L} / \mathrm{h}$ was administered intravenously (IV). Additionally, the gelding received $6 \mathrm{ml}$ flunixin meglumine $\left(50 \mathrm{mg} / \mathrm{ml}\right.$ Flunidol ${ }^{\circ}$ RPS $1.1 \mathrm{mg} / \mathrm{kg} \mathrm{BW}$ IV) ${ }^{4}$ for suspected abdominal pain. As the PCV dropped to $18 \%$ after the first infusion with $5 \mathrm{I} \mathrm{NaCl} 0.9 \%{ }^{3}$, the infusion was stopped.

The gelding was depressed overnight and showed no interest in food. Furthermore, the horse had a heart rate that varied between 60 and 64 beats/min. The respiratory rate stayed within normal limits. The oral mucus membranes remained pale, borborygmi were significantly reduced and the horse did not defecate.

The next day, ultrasonography of the abdomen confirmed further peritoneal effusion. The heart rate remained at 60 beats/ min and PCV had dropped to $12 \%$ (the number of erythrocytes to $3.65 \times 10^{6} / \mu \mathrm{L}$ ). Blood urea nitrogen (BUN) had increased slightly to $35 \mathrm{mg} / \mathrm{dL}$ (rr 10-24 mg/dL), and AST also increased slightly $(611 \mathrm{lU} / \mathrm{L}, \mathrm{rr}$ 226-366 IU/L). A high suspicion of progressive haemoperitoneum with an unknown cause was discussed with the owner, and diagnostic laparoscopy was advised.

\section{Surgery}

The horse was sedated with detomidine hydrochloride (Detogesic $\AA, 0.012 \mathrm{mg} / \mathrm{kg} \mathrm{IV})^{5}$ and butorphanol tartrate (Torbugesic $\left.V_{e t} \AA 0.025 \mathrm{mg} / \mathrm{kg} \mathrm{IV}\right)^{6}$ through a jugular catheter. The left and right flanks were clipped, and the gelding was restrained in stocks with its tail immobilized. After the surgical site was prepared with chlorhexidine and alcohol, the skin and underlying muscles were anaesthetized with $40 \mathrm{ml}$ of $2 \%$ lidocaine ( $2 \%$ lidocaine hydrochloride ${ }^{\circledR}{ }^{7}$ ) on both sides. The first portal for the laparoscope was made on the right side halfway between the last rib and the tuber coxae and just dorsal to the internal oblique abdominal muscle (Rijkenhuizen and Grinwis 1999). A $2 \mathrm{~cm}$ skin incision was made, and the muscles were bluntly divided with a Metzenbaum scissor. Then, a cannula with a trocar $\left(10 \mathrm{~mm}\right.$, Dr. Fritz $\left.{ }^{8}\right)$ was inserted in a caudoventral direction. After confirming the cannula was intraabdominally positioned, the trocar was replaced by the laparoscope (Storz', length $57 \mathrm{~cm}, 30$ degrees). The abdomen was insufflated with $\mathrm{CO}_{2}(1.6 \mathrm{~L} / \mathrm{min})$ until a maximum of $6 \mathrm{~mm} \mathrm{Hg}$ was reached. A second portal for a $5 \mathrm{~mm}$ trocar (Dr. Fritz $\left.{ }^{8}\right) 4 \mathrm{~cm}$ caudal and $6 \mathrm{~cm}$ distal to the laparoscopic portal was created under visualization.

The right dorsal abdominal cavity was inspected. The surface of the intestine was pale, and the motility was slightly reduced. The caecum was filled with gas, and between the caecum and abdominal wall, a large amount of blood was present. A suction drain attached to a suction pump was inserted through the second portal, and $10 \mathrm{~L}$ of unclotted blood was retrieved. The bloated caecum prevented the visualisation of the liver. After decompression of the caecum was performed, which used an 18-gauge laparoscopic needle, the caudate lobe of the right liver was examined, had a pale yellow irregular surface with sharp edges and was atrophic (Fig. 2). Directly cranial and lateral to the caudate lobe, the caudolateral pole of the right liver lobe became visible, which was very swollen due to subcapsular haematoma. The ventral portions of the liver 
and haematoma were underneath the fluid level and thus, could not be inspected.

Portals were created in the same manner on the left side of the abdomen. As on the right side, the surface of the intestine appeared pale, and the bladder was slightly filled. Besides haemoperitoneum and a swollen pale liver, no abnormalities were found in the left dorsal aspect of the abdomen (Fig. 3). After $25 \mathrm{~L}$ bloody fluid was evacuated with a suction drain, the liver came into view; it was swollen with a shiny pale colour.

A high suspicion of a ruptured liver or subcapsular haematoma on the right side as the cause of the haemoperitoneum was discussed with the owners. Based on the assumed diagnosis, the persistent blood loss and the inability to stop the bleeding, the owner elected euthanasia. The gelding was euthanized, and the body was submitted for necropsy.

\section{Pathology}

The pathological examination revealed the following abnormalities:

1. Hemoperitoneum with 8-10 L nonclotted blood.

2. A swollen, fragile liver with intrahepatic and subcapsular haematomas. Rupture of the liver capsule was present on various sections of the right lobe ventrally, which were up to $30 \mathrm{~cm}$ long and from which the ruptures had bled into the abdominal cavity.

3. Anaemic organs (heart, kidney, intestine) in the cadaver.

Histologically, a remarkably strong deposition of an eosinophilic, homogeneous, and hyaline material between atrophic liver cells and signs of amyloidosis were detected. A mild-to-moderate, subacute-to-chronic, mostly non-purulent pericholangitis/periportal hepatitis with a marked tendency to hepatocellular atrophy was present, especially in the area of amyloid-suspicious deposits. Congo red staining confirmed the suspicion of a liver amyloidosis showing characteristic salmon pink amorphous material.

The histopathological examination of the proliferation in the back showed predominantly parts of a chronically active

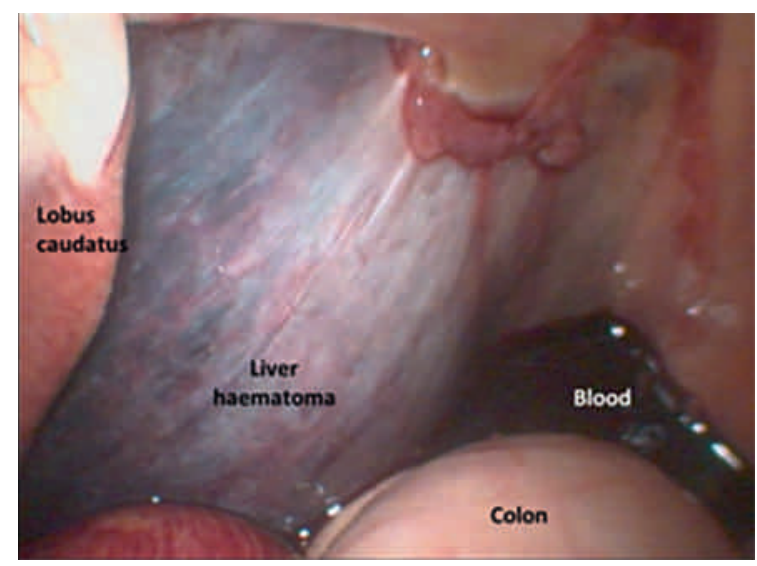

Fig. 2 Laparoscopic view of the cranial abdomen on the right side with the caudal lobe of the liver on the left side, the liver haematoma in the middle and free blood floating in the abdominal cavity. granulation-tissue organization with significant proportions of haemosiderosis and blood pigment deposits and centrally, a chronically active, purulent necrotizing inflammatory reaction, partly with a tendency towards abscessation. The findings confirmed the presence of organized haematoma with central abscessation.

\section{Discussion}

Spontaneous rupture of the liver is an exceptional phenomenon in horses not involved in serumproduction. In literature on human studies, spontaneous rupture of the liver is associated with pregnancy and HELLP syndrome or other liver pathology (Mascarenhas et al. 2002). In this case, ruptured capsule of the liver or subcapsular haematoma was highly suspected as being the cause of the haemoperitoneum, and this was indicated after laparoscopy and then confirmed by necropsy. Because of the amyloidosis, the tissue of the liver was very fragile, causing intrahepatic rupture and bleeding. The resulting subcapsular haematomas led to spontaneous ruptures, causing haemoperitoneum. Diagnosing spontaneous liver rupture is often difficult in the horse. First, the haemoperitoneum should be detected, followed by localization of the bleeding and its primary cause. For characterization of the origin of the bleeding, diagnostic laparoscopy or laparotomy are the most commonly used modalities in equines, whereas CT, MRI and/or angiography are the most helpful in humans (Lucey et al. 2005, Lubner et al. 2007). It is also necessary to ascertain if there is still active bleeding and whether the bleeding is slowing and might stop spontaneously. Surgery in this specific case was postponed to the next morning in order to stabilize the horse and observe the progression of the slowing intraabdominal bleeding.

Amyloidosis is an extrahepatocellular deposition process that damages liver cells as a secondary effect. Histologically, an amyloid appears as a homogeneous mass between the sinusoid wall and the liver cell bars. The deposits consist of an eosinophilic, insoluble substance. Amyloid comprises a group of proteins of different origins with a common property: they can be stained with Congo red, which is exhibited as apple-green birefringence on polarization microscopy. Amyloid proteins have a certain proportion of beta-sheet structure, which promotes the aggregation of several molecules and first leads to amyloid filaments, then to amyloid fibrils (van Andel

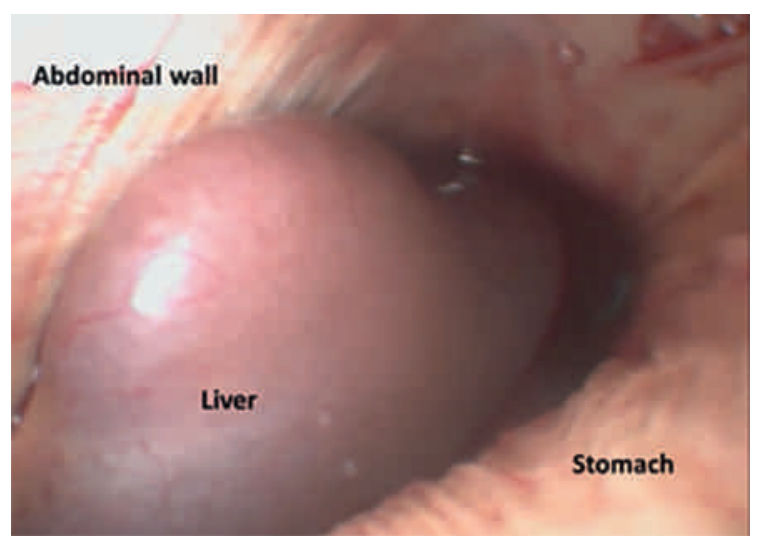

Fig. 3 Laparoscopic view of the left side of the abdomen with swollen liver. 
et al. 1988). These amyloid fibrils are not degradable. Liver cell bars become atrophic due to nutritional disorders, and pressure and can lead to intrahepatic haemorrhaging with eventual capsular rupture. The structure of the amyloidosis in the case described here was uncertain and could not be further investigated with the available histological methods. However, AA amyloidosis was suspected based on the histological appearance.

In humans several forms of AA amyloidosis have been described: generalized secondary amyloidosis, familial amyloidosis, and localized amyloidosis (Chen et al. 2010). Possible causes of hepatic AA amyloidosis include chronic inflammation or tumours. The AA-type precursor protein is serum amyloid $A$, an acute-phase protein in horses formed in the liver (Vanhooser et al. 1988). Serum amyloid A is subjected to proteolysis by enzymes in macrophages or by enzymes in the serum, resulting in the formation of amylogenic fibrils. Short-term inflammation induces the macrophages to release inflammatory mediators, such as interleukins 1 and 6 and tumour necrosis factor. Because the mediators of the cells in the liver release serum amyloid A, which is proteolytically cleaved, the formation of the amyloidogenic fibrils ensues (Gillmore et al. 2001, Simons et al. 2013). It is plausible that the inflammatory process on the back of the horse was the trigger to the generalized amyloidosis. In human AA amyloidosis is typically preceded by many years of an active inflammatory disease, and the clinical presentation is then relatively acute, suggesting a slow or long-delayed priming process that eventually triggers amyloid deposition (Simons et al. 2013). Circumferential proliferation in the rear of the horse caused by an organized haematoma with centrally purulent inflammatory granulation tissue could have underwent a slow process that triggered generalized amyloidosis with deposition in the liver, as the mass was recognized for several years. From a retrospective view, it might have been useful to remove the mass when the horse presented in the clinic for the first time. However, the poor healing properties of an incisional wound at this location led to a conservative approach.

Although the authors hypothesize that the subcutaneous mass in the back might have been the trigger for liver amyloidosis, the amyloidosis itself might have stimulated the formation of the swelling by reducing the synthesis of coagulation factors (Johns and Sweeney 2008). This seems less likely, as since the first bump was noticed, the owners did not notice sudden swelling, bleeding or signs of hypocoagulation in the horse, which could have occurred if coagulation had been insufficient. It remains unclear whether the mass in the back was a trigger or result of the amyloidosis.

The extent of amyloidosis in the liver was significant, and severe dysfunction of liver metabolism was likely, although blood analysis showed only slight changes. It is known that significant hepatic disease must be present before alterations in blood are apparent (Durham et al. 2010). In this patient, only aspartate aminotransferase (AST) was slightly increased. While all cells contain AST, skeletal muscle and liver cells exhibit the highest activity of this enzyme. The AST value is only useful to detect liver problems when it is used in combination with other liver-specific enzymes (serum gamma-glutamyl transferase, glutamate dehydrogenase, and serum sorbitol dehydrogenase) (Divers and Barton 2018). In our patient, these enzymes were within normal limits. An increase in the value of AST can also occur falsely in haemolysis, as erythrocytes are an important source of AST. Haemolysis did not occur in our patient. The hypoproteinaemia measure of $48 \mathrm{~g} /$ $\mathrm{dL}$ could be a sign of chronic liver disease (Divers and Barton 2018). Percutaneous liver biopsy might have been a helpful diagnostic procedure; however, has the risk of a bleeding complication, which was considered dangerous in our patient. Furthermore, with the acute bleeding of unknown cause it would not have been justified to wait two days for the results of the histological examination.

Unfortunately, histopathology of organs other than the liver was not performed, and it is unknown whether other organs were involved.

During laparoscopy, blood loss from a subcapsular liver haematoma was seen, and the liver rupture was considered to be the cause of the haemoperitoneum. To confirm the suspected diagnosis, it would have been possible to perform laparoscopy under general anaesthesia to explore the ventral side of both the right and left lobes of the liver. However, based on the laparoscopic findings, the cause of the haemoperitoneum was clear and no surgical or conservative options were available to treat the horse, and due to the continuous blood loss, it was decided that the best action to take was humane euthanasia.

Intraabdominal bleeding occurs in several forms ranging from arterial spurting to venous oozing (Dechant et al. 2006). In the current case, bleeding was persistent, and although it slowly stopped, it did not stop spontaneously. Because of pathological changes in the liver due to amyloidosis, it is assumed that just the sinusoids had bled, causing intrahepatic and subcapsular haematoma. Initially, the strong capsule of the liver has a tamponade effect; however, if pressure increases sufficiently, then rupture can occur ( $N g e n e$ et al. 2015, Yu et al. 2016). Furthermore, coagulopathy due to amyloidosis and/or blood loss could develop, stimulating the continuous bleeding. Unfortunately, no coagulation profile was performed because the progressive abdominal discomfort led to the decision to perform an emergency diagnostic laparoscopy that revealed the suspected cause of the haemoperitoneum.

Bleeding from a ruptured liver is extremely difficult to stop. Liver rupture in humans is often a fatal complication resulting from trauma, pregnancy, anticoagulant therapy, connective tissue disorders, liver infiltrative diseases, graft-versus-host disease, and hepatocellular carcinomas (Tam et al. 2009). Additionally, hepatic parenchymal and capsular infiltration with amyloids have been implicated as contributing factors in liver rupture (Tam et al. 2009). There are various methods to treat liver ruptures in humans; however, the preferred method of treatment for hepatic rupture is surgical intervention (Chen et al. 2002), and transcatheter hepatic artery embolization is described as being an effective procedure (Naito et al. 2008). Laparotomy or laparoscopy can be chosen to perform a partial hepatectomy, and gauze packing or mesh wrapping of the rupture can stop the bleeding and stabilize the patient (Ngene et al. 2015, Yu et al. 2016). Theoretically, explorative laparoscopy under general anaesthesia in the left lateral or even 
dorsal recumbency might have given a view on the rupture side, but this was not considered based on the poor prognosis of the equine patient and the knowledge that bleeding from a ruptured liver is extremely difficult to stop, particularly in horses, in which abdominal packing with swabs or mesh is hardly possible due to the size of the abdominal cavity.

\section{Conclusion}

Liver rupture as a cause of haemoperitoneum is an extremely rare complication of generalized AA amyloidosis in horses and should be considered in the differential diagnosis of haemoperitoneum. Laparoscopy in the standing horse as a diagnostic method to detect the origin of a haemoperitoneum should always be considered, as it can be performed quickly and is less dangerous for the horse than placing the horse under general anaesthesia.

\section{Manufacturer information}

' Boehringer Ingelheim, Viersen, Germany

$2+7$ bela-pharm, Vechta, Germany.

${ }^{3}$ Braun, Melsungen, Germany

$4+5+6$ cp-pharma, Burgdorf, Germany

${ }^{8}$ Dr. Fritz, Tuttlingen, Germany

${ }^{9}$ Storz, Tuttlingen, Germany

\section{References}

Chen Y. S., Qing-Hui Q., Zuo-Liang D. (2002) Etiology and management of hemorrhage in spontaneous liver rupture: a report of 70 cases. World J. Gastroenterol. 8, 1063-1066

Chen Y. S., Li W. Y., Ho, C. Y. (2010) Localized Nasopharyngeal Amyloidosis. J Chin Med Assoc 73, 549-552

Conwell R. C., Hillyer M. H., Mair T. S., Pirie R. S., Clegg P. D. (2010) Haemoperitoneum in horses: a retrospective review of 54 cases. Vet. Rec. 167, 514-518; DOI 10.1136/vr.c4569

Dechant J. E., Nieto J. E., Le Jeune S. S. (2006) Hemoperitoneum in horses: 67 cases (1989-2004). J. Am. Vet. Assoc. 229, 253-258; DOI 10.2460/javma.229.2.253

Divers T. J., Barton M. H. (2018) Chapter 14: Disorders of the Liver, Equine Internal Medicine, 4th edn., Ed: Reed, S.M., Bayly, W.M. and Sellon D.C. St. Louis. pp. 843-887

Durham A. E., Newton J. R. Smith K. C. Hillyer M. H., Hillyer L. L. Smith M. R. W., Marr, C. M. (2010) Retrospective analysis of historical, clinical, ultrasonographic, serum biochemical and haematological data in prognostic evaluation of equine liver disease. Equine Vet. J. 35, 542-547; DOI 10.2746/042516403775467324

Gatewood D. M., Douglass J. P., Cox J. H., DeBowes R. M., Kennedy G. A. (1990) Intra-abdominal hemorrhage associated with a granulosa-thecal cell neoplasm in a mare. J. Am. Vet. Med. Assoc. 196, 1827-1828

Gillmore J. D., Lovat L. B., Persey M. R., Pepys M. B., Hawkins P. N. (2001) Amyloid load and clinical outcome in $A A$ amyloidosis in relation to circulating concentration of serum amyloid A protein. Lancet 358, 24-29; DOI 10.1016/s0140-6736(00)05252-1
Green S. L., Specht T. E., Dowling S. C. Nixon A. J., Wilson J. H., Carrick J. B. (1988) Hemoperitoneum caused by rupture of a juvenile granulosa cell tumor in an equine neonate. J. Am. Vet. Med. Assoc. 193, 1417-1419

Hawkins J. F. (2016) Diagnosis, management and prognosis for haemoperitoneum in the horse. Equine Vet. Educ. 28, 364-366; DOI 10.111/i.1939-1676.2007.0006.x

Johns C., Sweeney R. W. (2008) Coagulation Abnormalities and Complications after Percutaneous Liver Biopsy in Horses. J. Vet. Intern. Med. 22, 185-189; DOI 10.1111/ן.1939-1676.2007.0006.x

Klohnen A. (2012) Evaluation of horses with signs of acute and chronic abdominal pain. In: Advances in Equine Laparoscopy, Ed: Ragle, C. A., Wiley Blackwell, Danvers, Massachusetts 93-1 18

Lubner M., Menjas C., Rucker C., Bhalla S., Peterson C. M., Wang L., Gratz B. (2007) Blood in the belly: CT findings of hemiperitoneum. Radiographics 27, 109-125

Lucey B. C., Varghese J. C., Soto J. A. (2005) Spontaneous hemoperitoneum: causes and significance. Curr. Probl. Diagn. Radiol. 34, 182195; DOI 10.1067/j.cpradiol.2005.06.001

Mascarenhas R., Mathias J., Varadarajan R., Geoghegan J., Traynor O. (2002) Spontaneous hepatic rupture. A report of five cases. HPB (Oxford) 4, 167-170; DOI 10.1080/13651820260503819

Naito K. S., Ichiyama T., Kawakami S., Kadoya M., Tabata T., Matsuda M., Ikeda S. (2008) AL amyloidosis with spontaneous hepatic rupture: successful treatment by transcatheter hepatic artery embolization. Amyloid 15, 137-139; DOI 10.1080/13506120802006187

Ngene N. C., Amin N., Moodley J. (2015) Ruptured subcapsular hematoma of the liver due to preeclampsia presenting as interstitial pregnancy and the role of intra- abdominal packing. Niger. J. Clin. Pract. 18, 300-303; 10.4103/1119-3077.151074

Pusterla N., Fecteau M. E., Madigan J. E., Wilson W. D., Magdesian K. G. (2005) Acute hemoperitoneum in horses: A review of 19 cases (1992-2003). J. Vet. Intern. Med. 19, 344-347; 10.1111/j.19391676.2005.tb02705.x

Rijkenhuizen A. B. M., Grinwis G. C. M. (1999) Castration of the stallion: Preferably in the standing horse by laparoscopic techniques? Pferdeheilkunde 16, 425-429.; DOI 10.21836/PEM19990504

Simons P. J., Al-Shawi R., Ellmerich S., Speck I., Aslam S., Hutchinson W. L., Mangione P. P., Disterer P., Gilbertson J. A., Hunt T., Millar D. J., Minogue S., Bodin K., Pepys M. B., Hawkins P. N. (2013) Pathogenetic mechanisms of amyloid A amyloidosis. Proc. Natl.. Acad. Sci. USA 110, 16115-16120; DOI 10.1073/pnas.1306621110

Tam M., Seldin D. C., Forbes B. M., Forbes B. M., Connors L. H., Skinner M., Oran B., Quillen K., Sanchorawala V. (2009) Spontaneous rupture of the liver in a patient with systemic AL amyloidosis undergoing treatment with high- dose melphalan and autologous stem cell transplantation: A case report with literature review. 16, 103-107; DOI 10.1080/13506120902879574

van Andel A. C., Gruys E., Kroneman J. (1988) Amyloid in the horse: A report of nine cases. Equine Vet. J. 20, 277-285; DOI 10.1111/ j.2042-3306.1988.tb01524.x

Vanhooser C. R., Reinemeyer C. R., Held J. P. (1988) Hepatic AA amyloidosis associated with severe strongylosis in a horse. Equine Vet. J. 20, 274-276; DOI 10.1111/i.2042-3306.1988.tb01522.x

Yu W. Y., Li Q. J., Gong J. P. (2016) Treatment strategy for hepatic trauma. Chin. J. Traumatol. 19, 168-171; DOI 10.1016/i.cjtee.201509.011 